\title{
WEAK THEORIES OF NONSTANDARD ARITHMETIC AND ANALYSIS
}

\author{
JEREMY AVIGAD
}

\begin{abstract}
A general method of interpreting weak higher-type theories of nonstandard arithmetic in their standard counterparts is presented. In particular, this provides natural nonstandard conservative extensions of primitive recursive arithmetic, elementary recursive arithmetic, and polynomial-time computable arithmetic. A means of formalizing basic real analysis in such theories is sketched.
\end{abstract}

§1. Introduction. Nonstandard analysis, as developed by Abraham Robinson, provides an elegant paradigm for the application of metamathematical ideas in mathematics. The idea is simple: use model-theoretic methods to build rich extensions of a mathematical structure, like second-order arithmetic or a universe of sets; reason about what is true in these enriched structures; and then transfer the results back to the ordinary mathematical universe. Robinson showed that this allows one, for example, to provide a coherent and consistent development of calculus based on the use of infinitesimals.

From a foundational point of view, it is natural to try to axiomatize such nonstandard structures. By formalizing the model-theoretic arguments, one can, in general, embed standard mathematical theories is conservative, nonstandard extensions. This was done e.g. by Kreisel, for second-order arithmetic [29]; Friedman, for first-order Peano arithmetic (unpublished); Nelson, for set theory [31]; and Moerdijk and Palmgren for intuitionistic first-order Heyting arithmetic [30] (see also [7]).

In recent years there has also been an interest in formalizing parts of mathematics in weak theories, at the level of primitive recursive arithmetic $(P R A)$, or below. The underlying motivations vary. One may be drawn by the general philosophical goal of minimizing ontological commitments, or, less ethereally, by the sport of seeing how little one can get away with. Alternatively, one may be interested in extracting additional mathematical information from standard mathematical developments (e.g. [24, 25, 27, 28]), or narrowing the theoretical

1991 Mathematics Subject Classification. 03B30,03F35,03F60,26E35.

Version: September 2, 2002. To appear in Stephen Simpson, editor, Reverse Mathematics 2001. Submitted September 2000, revised January 2001. Appendix A was added in July, 2001; Appendix B was added in August, 2002.

Partially supported by NSF grant DMS-0070600. Meeting

(c) 1000, Association for Symbolic Logic 1 
gap between abstract mathematics and concrete computation. In any event, a number of appropriate formal frameworks have been developed, including subsystems of first- and second-order arithmetic (e.g. [10, 35, 17, 18]), theories of finite types (e.g. [26, 28]), and versions of Feferman's theories of explicit mathematics (e.g. [39]), to name just a few.

The present work lies at the intersection of the two traditions just described, proposing the use of higher-type theories of nonstandard arithmetic as yet another framework for formalizing mathematics in weak theories. This combination seems promising, on a number of fronts. Work by Chuaqui, Sommer, and Suppes $[14,37,38]$ suggests that a good deal of ordinary analysis can be carried out in a theory of nonstandard analyis that is strictly weaker than $P R A$, and Tanaka [40] shows that nonstandard analysis can be developed in the theory $W K L_{0}$, which is conservative over PRA. Nelson [32] develops measuretheoretic probability using nonstandard arithmetic, though the presentation is informal and no axiomatic system is presented. Nonstandard methods have also been used in proof complexity and circuit complexity $[1,45,46]$; the theories presented here may provide a natural framework for the metamathematical analysis of such arguments. Aspects of constructive nonstandard analysis, described in [33], may carry over to weak theories as well.

In Section 2, I present a nonstandard higher-type extension of primitive recursive arithmetic, state the sense in which it is conservative over $P R A$, and provide a short model-theoretic proof. Section 3 provides another proof, via an explicit interpretation of the nonstandard higher-type theory in its standard counterpart. Both the model-theoretic proof and the interpretation are quite general, and apply to a wide range of weak theories of arithmetic; Section 4 explores variations, extensions, and applications of the central ideas. In Section 5, I discuss ways in which one can develop parts of real analysis in nonstandard theories like the ones considered here.

This paper constitutes a rough and exploratory proposal. In Section 6, I discuss directions for future research.

§2. A nonstandard extension of primitive recursive arithmetic. In this section I will describe a finite-type version of $P R A$ and a nonstandard extension thereof. For a fuller treatment of theories of finite type, see, for example, $[6,15,16,26,28,41,42,43,44]$. The theory called $P R A^{\omega}$ below is essentially a restriction of Gödel's theory $T$ with "predicative" recursors only, and with type 0 equality taken as basic. ${ }^{1}$

The set of primitive recursive functions is the smallest set of functions of various arities from the natural numbers to the natural numbers, containing the constant zero, projections, and the successor function, and closed under composition and primitive recursion. The language of $P R A$ has a symbol for each

\footnotetext{
${ }^{1}$ This theory is denoted $\widehat{T}_{0}$ in [6], and is the predicative restriction of the theory denoted $T_{0}$ in [43].
} 
primitive recursive function, and the axioms of $P R A$ consist of quantifier-free defining equations for these functions and a schema of induction for quantifierfree formulae.

Identifying relations with their characteristic functions, one can use primitive recursion to define the relation $x<y$; or, equivalently, one can add a relation symbol to the language of $P R A$ with appropriate defining equations. The schema of induction is equivalent to

$$
\forall x(\varphi(0) \wedge \forall y<x(\varphi(y) \rightarrow \varphi(y+1)) \rightarrow \varphi(x))
$$

where $\varphi$ is quantifier-free, possibly with parameters other than the one shown. Since the primitive recursive relations are closed under boolean operations and bounded quantification, the formula above is equivalent, in $P R A$, to a universal one. This fact can be used to show that PRA has a universal set of axioms. By Herbrand's theorem, it does not matter whether one takes the underlying logic to be first-order logic, or just the quantifier-free fragment: if the firstorder version of $P R A$ proves $\forall x \exists y \varphi(x, y)$ for $\varphi$ quantifier-free, then there is a function symbol $f$ and a propositional proof of $\varphi(x, f(x))$ from substitution instances of the universal axioms and axioms of equality.

The finite types are generated inductively as follows: $\mathrm{N}$ is a finite type (denoting the natural numbers, in the intended interpretation); and if $\sigma$ and $\tau$ are types, so are $\sigma \times \tau$ and $\sigma \rightarrow \tau$ (denoting the cross product of $\sigma$ and $\tau$ and the set of functions from $\sigma$ to $\tau$, respectively, in the full set-theoretic interpretation). I will take the simply-typed lambda calculus to have variables of all finite types, and constants denoting pairing functions, $\langle x, y\rangle$, and projections, $(z)_{0}$ and $(z)_{1}$, at all types. The set of lambda terms is further closed under lambda abstraction, denoted $\lambda x t$, and application, denoted $t(s)$. I will identify terms that differ up to a renaming of their free variables. If $t$ and $s$ are terms and $x$ is a variable of the appropriate type, then $t[s / x]$ denotes the result of substituting $s$ for $x$ in $t$, renaming bound variables if necessary. If I introduce a term as $t[x]$, then $t[s]$ abbreviates $t[s / x]$. I will write $t\left(s_{1}, \ldots, s_{k}\right)$ for $\left(\left(t\left(s_{1}\right)\right)\left(s_{2}\right)\right) \ldots\left(s_{k}\right)$, and $\sigma, \tau \rightarrow \rho$ instead of $\sigma \rightarrow(\tau \rightarrow \rho)$. $\mathrm{N}$ is sometimes called type 0 , and a function of type $\mathrm{N}, \ldots, \mathrm{N} \rightarrow \mathrm{N}$ is said to be of type 1 .

One obtains a higher-type extension of primitive recursive arithmetic as follows. Start with a many sorted version of first-order predicate logic with a sort for each finite type, and an equality relation = at type $\mathrm{N}$ only. The terms are the terms of the simply-typed lambda calculus with the extra constants described below. The theory includes equality axioms corresponding to $\beta$ reduction; since we only have type $\mathrm{N}$ equality, these have to be expressed as schemata:

- $r[(\lambda x . t)(s)]=r[t[s / x]]$

- $r\left[(\langle x, y\rangle)_{0}\right]=r[x]$

- $r\left[(\langle x, y\rangle)_{1}\right]=r[y]$ 
where in each case $r[z]$ is a term of type $\mathrm{N}$, with $z$ is a variable of the appropriate type. Add a constant 0 of type $\mathrm{N}$ and a constant $S$ of type $\mathrm{N} \rightarrow \mathrm{N}$, with axioms

- $\neg S(x)=0$

- $S(x)=S(y) \rightarrow x=y$

Then add a constant symbol $R$ of type $\mathrm{N},(\mathrm{N}, \mathrm{N} \rightarrow \mathrm{N}), \mathrm{N} \rightarrow \mathrm{N}$. The idea is that $R(a, f)$ is the function defined by primitive recursion from $a$ and $f$; hence we have the defining axioms

- $R(a, f, 0)=a$

- $R(a, f, S(x))=f(x, R(a, f, x))$

For each type $\sigma$ add a constant $\operatorname{Cond}_{\sigma}: \mathrm{N}, \sigma, \sigma \rightarrow \sigma$ with defining axioms

- $r[\operatorname{Cond}(0, x, y)]=r[x]$

- $r[\operatorname{Cond}(S(z), x, y)]=r[y]$

for type $\mathrm{N}$ terms $r[z]$ with $z$ of type $\sigma$. Finally, add a schema of quantifier-free induction, similar to the one for $P R A$. Call the resulting theory $P R A^{\omega}$.

Using the recursor, $R$, one can define all the primitive recursive functions. If we identify function symbols of $P R A$ with their definitions in $P R A^{\omega}, P R A$ is included in $P R A^{\omega}$. Conversely, we have the following:

TheOREM 2.1. PRA $A^{\omega}$ is a conservative extension of PRA.

A proof is sketched in [6, Section 5.1]; a similar proof, in the context of polynomial-time computable arithmetic, is found in [15].

I will now describe a nonstandard version of $P R A^{\omega}$, which I will denote $N P R A^{\omega}$. First, add a new predicate symbol $s t(x)$ to the language, with argument ranging over the natural numbers, and a new constant $\omega$ of type N. The predicate $s t$ is intended to denote the "standard" natural numbers, while $\omega$ is intended to denote a nonstandard natural number. Quantifiers ranging over the standard numbers are obtained by defining $\forall^{s t} x \varphi$ to be $\forall x(s t(x) \rightarrow \varphi)$ and $\exists^{s t} x \varphi$ to be $\exists x(s t(x) \wedge \varphi)$. A formula $\varphi$ is said to be internal if it does not involve st, and external otherwise.

To obtain $N P R A^{\omega}$, add the following axioms to $P R A^{\omega}$ :

- $\neg s t(\omega)$

- $s t(x) \wedge y<x \rightarrow s t(y)$

- $s t\left(x_{1}\right) \wedge \ldots \wedge s t\left(x_{k}\right) \rightarrow \operatorname{st}\left(f\left(x_{1}, \ldots, x_{k}\right)\right)$, for each type 1 term $f$ with no free variables and no occurence of $\omega$

In particular, the last axiom schema implies that the standard part of the universe is closed under the primitive recursive functions. In addition, add the following schema of $\forall$-transfer without parameters:

$$
\text { - } \forall^{s t} \vec{x} \psi(\vec{x}) \rightarrow \forall \vec{x} \psi(\vec{x})
$$

where $\psi$ is a quantifier-free internal formula that does not involve $\omega$, in which the only free variables are the type $\mathrm{N}$ variables shown. 
TheOREM 2.2. Suppose NPRA $A^{\omega}$ proves $\forall^{s t} x \exists y \varphi(x, y)$, where $\varphi$ is quantifierfree in the language of PRA with the free variables shown. Then $P R A^{\omega}$ proves $\forall x \exists y \varphi(x, y)$, and hence PRA proves it as well.

Since $\forall^{s t} x \exists y \varphi(x, y)$ is implied by both $\forall x \exists y \varphi(x, y)$ and $\forall^{s t} x \exists^{s t} y \varphi(x, y)$, we have:

Corollary 2.3. NPRA $A^{\omega}$ is a $\Pi_{2}$ conservative extension of PRA. Also, if $\varphi$ is quantifier-free in the language of $P R A$ and $N P R A^{\omega}$ proves $\forall^{s t} x \exists^{s t} y \varphi(x, y)$, then PRA proves $\forall x \exists y \varphi(x, y)$.

The second part of the corollary indicates a general pattern of reasoning in nonstandard arithmetic, whereby one uses nonstandard numbers to prove theorems about the standard ones.

Let $N P R A$ be $P R A$ together with the restriction of the axioms above to the smaller language. Since $N P R A$ is included in $N P R A^{\omega}$, we have the following:

Corollary 2.4. NPRA is conservative over PRA, in the sense of Theorem 2.2.

Corollary 2.4 has an easy model-theoretic proof, as follows. Suppose $P R A$ does not prove $\forall x \exists y \varphi(x, y)$. Let $L$ be the language of $P R A$, and let $c, d$, and $\omega$ be new constants. Let $T$ be the set of sentences containing all the following:

- The axioms of $P R A$

- $\forall y \neg \varphi(c, y)$

- $d>c$

- $\exists \vec{y} \psi(\vec{y}) \rightarrow \exists \vec{y}<d \psi(\vec{y})$, for each quantifier-free formula $\psi$ of $L$ with only the free variables shown

- $\omega>t$, for each closed term $t$ of $L+d$

Every finite subset of $T$ is consistent, since in any model of $P R A$ satisfying $\{\exists x \forall y \neg \varphi(x, y)\}$ we can choose an interpretation of $c$ satisfying $\forall y \neg \varphi(c, y)$, an interpretation of $d$ greater than finitely many witnesses for formulae of $L$ of the form $\exists \vec{y} \psi(\vec{y})$, and an interpretation of $\omega$ greater than the denotation of finitely many terms $t$ involving only $d$. By compactness, let $\mathcal{M}$ be a model of $T$. Let $S$ be the set of elements of the universe of $\mathcal{M}$ bounded by a closed term involving only the constant $d$, i.e.

$$
S=\left\{a \in|\mathcal{M}| \mid \text { for some closed term } t \text { of } L+d, a<t^{\mathcal{M}}\right\} .
$$

The reader can check that $\mathcal{M}$ becomes a model of NPRA satisfying $\operatorname{st}(c)$ and $\forall y \neg \varphi(c, y)$ when one uses $S$ to interpret the predicate $s t$.

A straightforward modification of this argument provides a proof of Theorem 2.2. And, in fact, the argument is much more general, since it relies on very few of the specific features of $P R A$. In the next section, I will present another proof of Theorem 2.2, by giving an interpretation of $N P R A^{\omega}$ in $P R A^{\omega}$. Such an interpretation is interesting in its own right, since it yields an explicit translation, with a polynomial bound on the increase in proof length. In addition, it tells us that Theorem 2.2 can be proved in weak fragments of 
arithmetic. We will see in Section 4 that the interpretation is almost as general as the model-theoretic argument, and so both are widely applicable.

In comparison with other nonstandard theories, the nonstandard axioms above are fairly weak. I will discuss strengthenings briefly in Sections 4 and 6 . But Section 5 suggests that the axioms above are already sufficient to formalize an interesting portion of real analysis.

§3. The interpretation. The interpretation of $N P R A^{\omega}$ in $P R A^{\omega}$ uses a forcing argument, described entirely in the language of $P R A^{\omega}$. For similar forcing arguments, see $[2,3,5,7,9]$.

Let $L$ denote the (typed) language of $P R A^{\omega}$, and $L^{s t}$ denote the language of $N P R A^{\omega}$, i.e. $L$ together with an extra constant, $\omega$, and a new predicate, st $(x)$. Our first step is to translate terms of $L^{s t}$ to terms of $L$. Choose a type $\mathrm{N}$ variable, $\omega$, in the language of $L$, corresponding to the constant, $\omega$, of $L^{s t}$. Also, assign to each variable $x$ of type $\sigma$ in $L^{s t}$ a variable $\tilde{x}$ of type $\mathrm{N} \rightarrow \sigma$ in $L$. Finally, if $t\left[x_{1}, \ldots, x_{k}\right]$ is a term of $L$ with the free variables shown, let $\widehat{t}$ denote the term $t\left[\tilde{x}_{1}(\omega), \ldots, \tilde{x}_{k}(\omega)\right]$ of $L$, where the constant $\omega$ of $L^{s t}$ is also replaced by the corresponding variable of $L$.

The idea is that we are taking elements of the universe of $L^{s t}$ to be named by terms of $L$ that depend on a "generic" element, $\omega$. It is not hard to check that the axioms of $\beta$-reduction are preserved by the translation.

Conditions of the forcing relation are ternary relations, considered as elements of type $\mathrm{N}, \mathrm{N}, \mathrm{N} \rightarrow \mathrm{N}$. Intuitively, a condition $p$ is supposed to represent the assertion $\forall^{s t} u \forall v p(u, v, \omega)$, where $\omega$ is the generic nonstandard element. If $p$ and $q$ are conditions, define $p \preceq q$ to be the formula $\forall u, v, \omega(p(u, v, \omega) \rightarrow$ $q(u, v, \omega)$ ), read " $p$ is stronger than (or equivalent to) $q$." Note that if $p$ and $q$ are conditions, then their conjunction, $p \wedge q$, satisfies $p \wedge q \preceq p$ and $p \wedge q \preceq q$. Sometimes, if $p$ is a condition and $A$ is another ternary relation, I will write $p \wedge \forall^{s t} u \forall v A(u, v, \omega)$ instead of $p \wedge A$. This is nothing more than a useful convention that will keep us mindful of the informal interpretation of the conditions.

We are now ready to define a relation $p \Vdash \varphi$ between conditions $p$ and formulae $\varphi$ of $L^{s t}$. It will be convenient to take the logical connectives to be $\forall, \wedge, \rightarrow, \perp$. With this choice of connectives, $\neg \varphi$ abbreviates $\varphi \rightarrow \perp, \exists x \varphi$ abbreviates $\neg \forall x \neg \varphi$, and $\varphi \vee \psi$ abbreviates $\neg(\neg \varphi \wedge \neg \psi)$. The forcing relation is defined inductively, as follows:

1. $p \Vdash \perp \equiv \exists z \forall \omega \neg \forall u<z \forall v p(u, v, \omega)$.

2. $p \Vdash t_{1}=t_{2} \equiv \exists z \forall \omega\left(\forall u<z \forall v p(u, v, \omega) \rightarrow \widehat{t}_{1}=\widehat{t}_{2}\right)$.

3. $p \Vdash t_{1}<t_{2} \equiv \exists z \forall \omega\left(\forall u<z \forall v p(u, v, \omega) \rightarrow \widehat{t}_{1}<\widehat{t}_{2}\right)$.

4. $p \Vdash s t(t) \equiv \exists z \forall \omega(\forall u<z \forall v p(u, v, \omega) \rightarrow \widehat{t}<z)$.

5. $p \Vdash \varphi \rightarrow \psi \equiv \forall q \preceq p(q \Vdash \varphi \rightarrow q \Vdash \psi)$.

6. $p \Vdash \varphi \wedge \psi \equiv(p \Vdash \varphi) \wedge(p \Vdash \psi)$.

7. $p \Vdash \forall x \varphi \equiv \forall \tilde{x}(p \Vdash \varphi)$ 
If $\varphi$ is a formula in the language $L^{s t}$ with free variables $x_{1}, \ldots, x_{k}$, then $p \Vdash \varphi$ is a formula in the language $L$ with free variables $\omega, \tilde{x}_{1}, \ldots, \tilde{x}_{k}$, as well as $p$. Notice that we are allowing that some conditions force $\perp$. In the definition of $p \Vdash \varphi \rightarrow \psi$, the quantifier $\forall q \preceq p$ ranges over conditions. It is not difficult to show that $p \Vdash \varphi \rightarrow \psi$ is equivalent to $\forall q(q \Vdash \varphi \rightarrow p \wedge q \Vdash \psi)$; I will use both formulations of $p \Vdash \varphi \rightarrow \psi$ below. Define $\Vdash \varphi$ to be $\forall p(p \Vdash \varphi)$, read " $\varphi$ is forced."

The following informal considerations may help explain the motivation behind the definition of forcing at the atomic clauses. Think of a condition $p$ as representing an infinite set of sentences,

$$
\{\forall v p(0, v, \omega), \forall v p(1, v, \omega), \forall v p(2, v, \omega), \ldots\} .
$$

If we call this set $S_{p}$ then clause 2 , for example, asserts that $p$ forces $t_{1}=t_{2}$ if and only if $\widehat{t}_{1}=\widehat{t}_{2}$ is a consequence of a finite subset of $S_{p}$.

The proofs of the next five lemmata are routine and standard. (See, for example, $[3,9]$ for a little more detail.)

Lemma 3.1. Suppose $t$ and $s$ are terms of $L^{s t}, r[z]$ is a type $\mathrm{N}$ term of $P R A^{\omega}$, and $z$ has the same type as $t$. Then $P R A^{\omega}$ proves

$$
r[\widehat{t}[\lambda \omega \widehat{s} / \tilde{x}]]=r[\widehat{t[s / x]}] .
$$

Proof. By induction on $t$. Informally, in the base case where $t$ is $x$, we have

$$
\widehat{t}[\lambda \omega \widehat{s} / \tilde{x}]=\tilde{x}(\omega)[\lambda \omega \widehat{s} / \tilde{x}]=(\lambda \omega \widehat{s})(\omega)=\widehat{s}=\widehat{t[s / x]} .
$$

The other cases are easy.

Lemma 3.2 (substitution). For each formula $\varphi$ and term $s$ in the language of $L^{s t}, P R A^{\omega}$ proves $p \Vdash \varphi[s / x] \leftrightarrow(p \Vdash \varphi)[\lambda \omega \widehat{s} / \tilde{x}]$.

Proof. By induction on $\varphi$. Lemma 3.1 takes care of the base cases. $\dashv$

LEMma 3.3 (monotonicity). For each formula $\varphi$ of $L^{s t}$, PRA $A^{\omega}$ proves $p \Vdash$ $\varphi \wedge q \preceq p \rightarrow q \Vdash \varphi$.

Proof. Induction on $\varphi$.

Lemma 3.4. For each formula $\varphi$ in the language of $L^{s t}$, $P R A^{\omega}$ proves $\Vdash$ $(\perp \rightarrow \varphi)$.

Proof. Induction on $\varphi$.

Lemma 3.5. For each formula $\varphi$ in the language of $L^{\text {st }}$, if $\varphi$ is provable in intuitionistic logic, then $P R A^{\omega}$ proves $\Vdash \varphi$.

Proof. Induction on proofs.

Since we are interpreting the connectives $\vee$ and $\exists$ in terms of their doublenegation translations, in order to extend Lemma 3.5 to classical logic we only 
need to show that, under the forcing relation, each atomic formula is equivalent to its double-negation. For that purpose, it will be helpful to have the alternative characterizations of $p \Vdash s t(t)$ provided by the following lemma.

To make sense of clause 2 below, some additional considerations are needed. If $\varphi$ is a quantifier-free formula of $L$ involving $u, v$, and $\omega$, then, fixing the other free variables, $\varphi$ is provably equivalent to a ternary relation $p(u, v, \omega)$. In clause 2 , the condition " $\forall{ }^{s t} u(\widehat{t} \nless u)$ " should be interpreted as the ternary relation corresponding to $\widehat{t} \nless u$, which depends on $u$ and $\omega$, but not $v$. I will use similar conventions below without mentioning them explicitly.

LEMma 3.6. PRA ${ }^{\omega}$ proves that the following are pairwise equivalent:

1. $p \Vdash s t(t)$.

2. $p \wedge \forall^{s t} u(\widehat{t} \nless u) \Vdash \perp$.

3. $\exists y((p \Vdash t<x)[\lambda \omega y / \tilde{x}])$.

Also, $\forall^{s t} u(\widehat{t} \nless u) \Vdash \neg s t(t)$.

Regarding clause 3 , remember that in $p \Vdash t<x$ the free variable $x$ is replaced by $\tilde{x}(\omega)$. So 3 asserts that $t$ is standard (at $p$ ) if and only if it has a bound that does not depend on $\omega$.

Proof. The equivalences follow almost immediately from the definition of $p \Vdash s t(t)$, which is also easily seen to be equivalent to $\exists z, y \forall \omega(\forall u<$ $z \forall v p(u, v, \omega) \rightarrow \widehat{t}<y)$. For the last claim, note that $\forall^{s t} u(t \nless u) \Vdash \neg s t(t)$ is equivalent to the statement "for every $p, 1$ implies $2 . "$

Lemma 3.7. For each formula $\varphi$ of $L^{\text {st }}$, PRA $A^{\omega}$ proves $\Vdash \neg \neg \varphi \rightarrow \varphi$.

Proof. We will only verify this in the case that $\varphi$ is atomic. The general case follows by induction on formulae (working directly with the forcing definitions, or using intuitionistically valid inferences, which we already know are sound for forcing).

To handle the case where $\varphi$ is of the form $t_{1}=t_{2}$, we first have to observe that $P R A^{\omega}$ proves $\widehat{t}_{1} \neq \widehat{t}_{2} \Vdash \neg\left(t_{1}=t_{2}\right)$. To see this, remember that $\widehat{t}_{1} \neq \widehat{t}_{2} \Vdash$ $\neg\left(t_{1}=t_{2}\right)$ is equivalent to the assertion that whenever a condition $p$ forces $t_{1}=t_{2}, p \wedge \widehat{t}_{1} \neq \widehat{t}_{2}$ forces $\perp$; but verifying this is just a matter of unwinding definitions.

To see that $P R A^{\omega}$ proves $\Vdash \neg \neg\left(t_{1}=t_{2}\right) \rightarrow t_{1}=t_{2}$, argue (in $P R A^{\omega}$ ) as follows: suppose $p \Vdash \neg \neg\left(t_{1}=t_{2}\right)$. Then $p \wedge \widehat{t}_{1} \neq \widehat{t}_{2} \Vdash \perp$. Checking the definitions again, it is not hard to verify that this is equivalent to $p \Vdash t_{1}=t_{2}$, as required.

The same argument shows that $P R A^{\omega}$ proves $\Vdash \neg \neg t_{1}<t_{2} \rightarrow t_{1}<t_{2}$.

Finally, for the case that $\varphi$ is st $(t)$, argue in $P R A^{\omega}$, and suppose $p \Vdash \neg \neg s t(t)$. Then, by Lemma 3.6, $p \wedge \forall^{s t} u(\widehat{t} \nless u) \Vdash \perp$. By Lemma 3.6 again, $p \Vdash s t(t)$, as required.

Lemma 3.8. For each formula $\varphi$ in the language of $L^{\text {st }}$, if $\varphi$ is provable classically, then $P R A^{\omega}$ proves $\Vdash \varphi$. 
Proof. Follows from Lemmata 3.5 and 3.7.

We are now ready to consider the axioms of $N P R A^{\omega}$. We will soon see that all of the axioms other than $\neg s t(\omega)$ are forced. But first, we will need the following lemma. If $\varphi$ is any quantifier-free internal formula in the language of $L^{s t}$, let $\widehat{\varphi}$ denote the translation of $\varphi$ to the language of $L$, derived from the corresponding translation of terms. More explicitly, if $\varphi\left(x_{1}, \ldots, x_{k}\right)$ has the free variables shown, then $\widehat{\varphi}$ is the formula $\varphi\left(\tilde{x}_{1}(\omega), \ldots, \tilde{x}_{k}(\omega)\right)$, with the understanding that if the constant, $\omega$, occurs in $\varphi$, it is replaced by the variable, $\omega$, in $\widehat{\varphi}$.

LEMMA 3.9. For each quantifier-free internal formula $\varphi$ of $L^{s t}, P R A^{\omega}$ proves

1. $\widehat{\varphi} \Vdash \varphi$.

2. $p \Vdash \varphi$ is equivalent to $\exists z \forall \omega(\forall u<z \forall v p(u, v, \omega) \rightarrow \widehat{\varphi})$.

Proof. The two claims are proved simultaneously by induction on $\varphi$. If $\varphi$ is atomic, then 2 holds by definition and 1 is handled as in the proof of Lemma 3.7. Since the case where $\varphi$ is of the form $\theta \wedge \eta$ is straightforward, we only need to consider the case where $\varphi$ is of the form $\theta \rightarrow \eta$.

For 1 , we need to show $\widehat{\theta} \rightarrow \widehat{\eta} \Vdash \theta \rightarrow \eta$. So suppose $p \Vdash \theta$; we need to show $p \wedge(\widehat{\theta} \rightarrow \widehat{\eta}) \Vdash \eta$. By the induction hypothesis, it suffices to show

$$
\exists z \forall \omega(\forall u<z \forall v p(u, v, \omega) \wedge(\widehat{\theta} \rightarrow \widehat{\eta}) \rightarrow \widehat{\eta}),
$$

since $\widehat{\theta} \rightarrow \widehat{\eta}$ does not depend on $u$ or $v$. From the assumption $p \Vdash \theta$ and the inductive hypothesis we have

$$
\exists z \forall \omega(\forall u<z \forall v p(u, v, \omega) \rightarrow \widehat{\theta}),
$$

from which (1) follows.

For the forward direction of 2, Suppose $p \Vdash(\theta \rightarrow \eta)$. By the inductive hypothesis, we know $\widehat{\theta} \Vdash \theta$, and so $p \wedge \widehat{\theta} \Vdash \eta$. From the definition, it is easy to show that this implies

$$
\exists z \forall \omega(\forall u<z \forall v p(u, v, \omega) \rightarrow(\widehat{\theta} \rightarrow \widehat{\eta})) .
$$

For the other direction, suppose the last displayed formula holds, $q \preceq p$, and $q \Vdash \theta$. By the inductive hypothesis we have

$$
\exists z \forall \omega(\forall u<z \forall v q(u, v, \omega) \rightarrow \widehat{\theta})
$$

and hence

$$
\exists z \forall \omega(\forall u<z \forall v q(u, v, \omega) \rightarrow \widehat{\eta}) .
$$

By the inductive hypothesis, this is equivalent to $q \Vdash \eta$.

Lemma 3.10. If $\varphi$ is an axiom of $P R A^{\omega}$, then $P R A^{\omega}$ proves $\Vdash \varphi$.

Proof. All the axioms of $P R A^{\omega}$ are universal, which is to say, they are of the form $\forall x_{1}, \ldots, x_{k} \varphi\left(x_{1}, \ldots, x_{k}\right)$, where $\varphi$ is quantifier-free and internal. By Lemma 3.9, $\forall \forall \vec{x} \varphi$ is equivalent to $\forall \tilde{x}_{1}, \ldots, \tilde{x}_{k} \forall \omega \varphi\left(\tilde{x}_{1}(\omega), \ldots, \tilde{x}_{k}(\omega)\right)$. 
The axioms corresponding to $\beta$-reduction are easy to verify, and otherwise, the translation of each axiom follows immediately from the untranslated version.

LEMMA 3.11. For each constant term $f$ of type $\mathrm{N}^{k} \rightarrow \mathrm{N}$ of $L^{\text {st }}$ not involving $\omega, P R A^{\omega}$ proves $\Vdash \forall x_{1}, \ldots, x_{k}\left(s t\left(x_{1}\right) \wedge \ldots \wedge s t\left(x_{k}\right) \rightarrow f\left(x_{1}, \ldots, x_{k}\right)\right)$.

Proof. The key point is that if $f$ is as in the hypothesis, it does not depend on $\omega$. Argue in $P R A^{\omega}$. Suppose $p \Vdash\left(s t\left(x_{1}\right) \wedge \ldots \wedge s t\left(x_{k}\right)\right)$, i.e. $\exists z \forall \omega\left(\forall u<z \forall v p(u, v, \omega) \rightarrow\left(\tilde{x}_{1}(\omega)<z \wedge \ldots \wedge \tilde{x}_{k}(\omega)<z\right)\right)$. Letting $z^{\prime}=\max _{\vec{v}<z} \widehat{f}\left(v_{1}, \ldots, v_{k}\right)$, we have $\exists z, z^{\prime} \forall \omega(\forall u<z \forall v p(u, v, \omega) \rightarrow$ $\widehat{f}\left(\tilde{x}_{1}(\omega), \ldots, \tilde{x}_{k}(\omega)\right)<z^{\prime}$, which implies $p \Vdash s t\left(f\left(x_{1}, \ldots, x_{k}\right)\right)$.

Lemma 3.12. PRA $A^{\omega}$ proves $\Vdash \forall x, y(s t(x) \wedge y<x \rightarrow s t(y))$.

Proof. Argue in $P R A^{\omega}$. Suppose $p \Vdash s t(x)$ and $p \Vdash y<x$. Then $\exists z \forall \omega(\forall u<z \forall v p(u, v, \omega) \rightarrow \tilde{x}(\omega)<z)$ and $\exists z \forall \omega(\forall u<z \forall v p(u, v, \omega) \rightarrow$ $\tilde{y}(\omega)<\tilde{x}(\omega))$. Picking $z$ to be the maximum of any two witnesses to these statements, we have $\exists z \forall \omega(\forall u<z \forall v p(u, v, \omega) \rightarrow \tilde{y}(\omega)<z)$, which is $p \Vdash s t(y)$.

We have dealt with all the axioms except for $\forall$-transfer and $\neg s t(\omega)$. The next lemma deals with the former.

LEMMA 3.13. If $\varphi(\vec{x})$ is any quantifier-free formula of $L$ with only the type $\mathrm{N}$ variables shown, $P R A^{\omega}$ proves $\Vdash \forall^{s t} \vec{x} \varphi(\vec{x}) \rightarrow \forall \vec{x} \varphi(\vec{x})$.

Proof. For notational simplicity, let us assume that $\vec{x}$ is a single variable. Argue in $P R A^{\omega}$. Suppose $p \Vdash \forall^{s t} x \varphi(x)$. By Lemma 3.6, we have $\forall w((p \Vdash$ $\varphi(x))[\lambda \omega w / \tilde{x}])$. Since $\widehat{\varphi(x)}[\lambda \omega w / \tilde{x}]$ is equivalent to $\varphi(w)$, by Lemma 3.9 we have

$$
\forall w \exists z \forall \omega(\forall u<z \forall v p(u, v, \omega) \rightarrow \varphi(w)) .
$$

Since $\varphi$ does not depend on $\omega$ or $z$, this is equivalent to

$$
\exists z \forall \omega(\forall u<z \forall v p(u, v, \omega) \rightarrow \forall w \varphi(w)),
$$

which in turn implies

$$
\forall \tilde{x} \exists z \forall \omega(\forall u<z \forall v p(u, v, \omega) \rightarrow \varphi(\tilde{x}(\omega))) .
$$

But the last formula is equivalent to $p \Vdash \forall x \varphi(x)$, which is what we want. $\dashv$

LEMMA 3.14. Suppose $\varphi$ is any formula of $L^{s t}$, and $N P R A^{\omega}$ proves $\varphi$. Then $P R A^{\omega}$ proves $\forall^{s t} u(\omega \nless u) \Vdash \varphi$.

Proof. By Lemma 3.6, we have $\forall^{s t} u(\omega \nless u) \Vdash \neg s t(\omega)$, and we have shown that all the other axioms of $N P R A^{\omega}$ are forced.

We are now only one lemma away from the proof of the main theorem. 
LEMma 3.15. Suppose $\varphi\left(y, x_{1}, \ldots, x_{k}\right)$ is a quantifier-free internal formula of $L^{\text {st }}$ with the free variables shown, and $y$ is of type $\mathrm{N}$. Then PRA ${ }^{\omega}$ proves $\forall v \varphi\left(v, \tilde{x}_{1}(\omega), \ldots \tilde{x}_{k}(\omega)\right) \Vdash \forall y \varphi\left(y, x_{1}, \ldots, x_{k}\right)$.

Proof. Unwinding the definition and using Lemma 3.9, we see that we need to show that $P R A^{\omega}$ proves

$$
\forall \tilde{y} \exists z \forall \omega\left(\forall v \varphi\left(v, \tilde{x}_{1}(\omega), \ldots, \tilde{x}_{k}(\omega)\right) \rightarrow \varphi\left(\tilde{y}(\omega), \tilde{x}_{1}(\omega), \ldots, \tilde{x}_{k}(\omega)\right)\right) .
$$

But this is immediate.

Proof of Theorem 2.2. Suppose $N P R A^{\omega}$ proves $\forall^{s t} x \exists y \varphi(x, y)$ with $\varphi$ quantifier-free in the language of $L$, and argue in $P R A^{\omega}$. By Lemma 3.14, we have

$$
\forall^{s t} u(\omega \nless u) \Vdash \forall^{s t} x \exists y \varphi(x, y) .
$$

Let $w$ be arbitrary. Since $(p \Vdash s t(x))[\lambda \omega w / \tilde{x}]$, we have

$$
\left(\forall^{s t} u(\omega \nless u) \Vdash \exists y \varphi(x, y)\right)[\lambda \omega w / \tilde{x}] .
$$

Keep in mind that $\exists y \varphi(x, y)$ abbreviates $\neg \forall y \neg \varphi(x, y)$. By the previous lemma, $\forall v \neg \varphi(\tilde{x}(\omega), v) \Vdash \forall y \neg \varphi(x, y)$, so we have

$$
\left(\forall^{s t} u(\omega \nless u) \wedge \forall v \neg \varphi(\tilde{x}(\omega), v) \Vdash \perp\right)[\lambda \omega w / \tilde{x}],
$$

which expands to

$$
\exists z \forall \omega(\forall u<z \forall v(\omega \nless u \wedge \forall v \neg \varphi(w, v)) \rightarrow \perp) .
$$

This is classically equivalent to

$$
\exists z \forall \omega(\exists u<z(\omega<u) \vee \exists v \varphi(w, v)) .
$$

Given a $z$ witnessing this statement, choose $\omega=z$. Then we have $\forall u<z(\omega \nless$ $u$ ), and hence $\exists v \varphi(w, v)$. Since $w$ was arbitrary, we have $\forall w \exists v \varphi(w, v)$, as desired.

$\S 4$. Weak theories of nonstandard arithmetic. In this section, I will discuss variations of Theorem 2.2, and some applications. To start with, there are a number of features of Theorem 2.2 and its proofs that are worth noting.

The first has to do with the treatment of equality. The theories $P R A^{\omega}$ and $N P R A^{\omega}$ were presented with only equality at type $\mathrm{N}$ as a basic relation. Of course, one can define equality at higher types extensionally; for example, if $f$ and $g$ are of type $\mathrm{N} \rightarrow \mathrm{N}$ one can take $f=g$ to be $\forall x(f(x)=g(x))$. Doing so does not guarantee that the usual equality axioms, $f=g \rightarrow \varphi(f)=\varphi(g)$, follow. But, using a method due to Luckhardt, one can interpret a fully extensional version of $N P R A^{\omega}$ in our intensional version, by relativizing all quantifiers and variables to the "hereditarily extensional objects." This interpretation preserves $\Pi_{2}$ formulae (as well as $\forall^{s t} \exists^{s t}$ formulae, etc.). So Theorem 2.2 extends to extensional versions of $N P R A^{\omega}$ as well. For a discussion 
of some of the issues related to various treatments of equality, see [6, Section 3.1], [43, Section 3.1], and [15, Section 7].

Second, most of the higher types were not used by the intepretation in an essential way. It suffices to have a theory in which the types are closed under the operation $\sigma \mapsto(\mathrm{N} \rightarrow \sigma)$, so, for example, the interpretation works just as well for second-order versions of $N P R A$ and $P R A$, associating $k$-ary function variables of the first theory to $(k+1)$-ary function variables of the second.

Finally, very little reference was made to the specifics of $P R A^{\omega}$ itself. In the interpretation, only the following features came into play:

1. $P R A^{\omega}$ proves that $<$ is transitive and anti-reflexive, and satisfies the sentence $\forall x, y \exists z(x<z \wedge y<z)$.

2. $P R A^{\omega}$ has a universal set of axioms.

3. If $\varphi(x, y, z)$ is a quantifier-free formula, possibly with free variables shown, $P R A^{\omega}$ proves

$$
\exists R \forall x, y, z(\varphi(x, y, z) \leftrightarrow R(x, y, z)),
$$

where $R$ ranges over a suitable representation of ternary relations.

4. $P R A^{\omega}$ proves that the ternary relations are closed under conjunction.

5. If $f$ is a closed type 1 term, then $P R A^{\omega}$ proves $\forall z \exists w \forall \vec{x}<z(f(\vec{x})<w)$. (This was used in the proof of Lemma 3.11).

In fact, most of the proofs in the previous section required only the intuitionistic fragment of $P R A^{\omega}$. The proof of Lemma 3.13, which showed that the $\forall$-transfer schema without parameters is forced, used classical logic. But if one is willing to give up transfer, then only the final proof of Theorem 2.2 requires an inference that is not strictly intuitionistic, in the form of Markov's principle for quantifier-free formulae; and a slight rewriting of the forcing relation, along the lines of [3], can be used to render the argument entirely intuitionistic. On the other hand, if one has no qualms about the use of classical logic, the presentation of the forcing relation can be simplified; see Appendix B below.

In sum, both the model-theoretic argument sketched at the end of Section 2 and the syntactic interpretation of Section 3 generalize considerably. For example, let $P V$ be Cook's theory of polynomial-time computable functions, and let $P V^{\omega}$ be a corresponding higher-type generalization (i.e. the theory called $P V^{\omega}$ in [15], but with induction restricted to quantifier-free formulae; see also [6, Section 5.2]). Let $N P V^{\omega}$ be the nonstandard version obtained by adding the nonstandard axioms of Section 2. Then we have

TheOREM 4.1. $N P V^{\omega}$ is conservative over $P V^{\omega}$ and $P V$, in the sense of Theorem 2.2.

Similarly, let ERA denote elementary recursive arithmetic, obtained by adding,$+ \times$, and $x^{y}$ to $P R A$ but restricting the recursions to those that can be bounded by a term (see e.g. [4, 37, 38]). ERA is a conservative extension of the theory alternatively known as $E F A$, for "elementary function arithmetic", or $I \Delta_{0}(\exp )$ (see [21]). Let $E R A^{\omega}$ be the natural higher-type version of $E R A$ 
(similar to the theory $G_{3} A^{\omega}$ of [26, Section 2.2], but without the additional universal sentences in clause 9), and let $N E R A^{\omega}$ be the nonstandard version of $E R A^{\omega}$. Then we have

THEOREM 4.2. NERA ${ }^{\omega}$ is conservative over $E R A^{\omega}$ and ERA, in the sense of Theorem 2.2.

Similar versions of Theorem 2.2 hold, for example, for the theory denoted $T+(\mu)$ in [6], and for the theories $G_{n} A^{\omega}$ of [26].

The nonstandard axioms can have interesting consequences for the standard numbers. Recall that a formula is bounded, or $\Delta_{0}$, if all its quantifiers are bounded, and $\Sigma_{1}$ if it is of the form $\exists \vec{x} \varphi$, where $\varphi$ is bounded. Consider the collection principle, $B \Sigma_{1}$ :

$$
\forall x<z \exists y \varphi(x, y) \rightarrow \exists w \forall x<z \exists y<w \varphi(x, y)
$$

where $\varphi$ is $\Sigma_{1}$. Let $B \Sigma_{1}^{s t}$ denote the relativization of $B \Sigma_{1}$ to the standard numbers, where $z$ is assumed to be standard. The following proposition still holds even if $\varphi$ has additional parameters that are not necessarily standard.

Proposition 4.3. NERA $A^{\omega}$ and $N P R A^{\omega}$ prove $B \Sigma_{1}^{s t}$.

Proof. By pairing existential quantifiers, we may assume $\varphi$ is $\Delta_{0}$. Argue in $N E R A^{\omega}$ or $N P R A^{\omega}$. Suppose $z$ is standard and $\forall x<z \exists^{s t} y \varphi(x, y)$. Then for any nonstandard number $w$, we have $\forall x<z \exists y<w \varphi(x, y)$. Since, in $N E R A^{\omega}$ and $N P R A^{\omega}$, every bounded formula is equivalent to a quantifier-free (even atomic) one, by induction there is a least $w$ such that this last formula is satisfied. Since the nonstandard numbers are closed under predecessor, this least $w$ is standard.

Another interesting fact is that we can interpret the theory $W K L_{0}^{*}$ of Simpson and Smith $[35,36]$. This theory is equivalent to a second-order version of $E R A$ with set variables, together with a recursive comprehension axiom, $(R C A)$, and a weak version of König's lemma, $(W K L)$, which asserts that every infinite tree on $\{0,1\}$ has an infinite path. For details, see [35, 36].

THEOREM 4.4. $W K L_{0}^{*}$ is conservative over ERA for $\Pi_{2}$ formulae.

Proof. One can interpret $W K L_{O}^{*}$ in $N E R A$, interpreting the first-order universe as the standard numbers of $N E R A$, and interpreting the second-order universe as the standard parts of nonstandard finite sets of NERA. Here we are using the fact that in $N E R A$, one can code finite sets as natural numbers; note that if $\mathcal{M}$ is a model of NERA and $S$ is a set coded in $\mathcal{M}$, then the intersection of $S$ with the standard numbers of $\mathcal{M}$ may be unbounded.

Lemma IV.4.4 of [35] shows that $(W K L)$ and $(R C A)$ follow from a single schema of $\Sigma_{1}$ separation:

$$
\begin{aligned}
\forall x \neg(\exists y \varphi(x, y) \wedge & \exists z \psi(x, z)) \rightarrow \\
& \exists S \forall x((\exists y \varphi(x, y) \rightarrow x \in S) \wedge(\exists z \psi(x, z) \rightarrow x \notin S)),
\end{aligned}
$$


where $\varphi$ is $\Delta_{0}$. To see that this holds in the interpretation, argue in NERA. Suppose for every standard $x$ we have $\neg\left(\exists^{s t} y \varphi(x, y) \wedge \exists^{s t} z \psi(x, z)\right)$. Let $S$ be the finite set

$$
S=\{x<\omega \mid \exists y<\omega(\varphi(x, y) \wedge \forall z<y \neg \psi(x, z))\} .
$$

It is not hard check that for each standard $x$, we have

$$
\left(\exists^{s t} y \varphi(x, y) \rightarrow x \in S\right) \wedge\left(\exists^{s t} z \psi(x, z) \rightarrow x \notin S\right),
$$

as required.

The results of [36] are more general; for example, the first-order consequences of $W K L_{0}^{*}$ are exactly those of $E R A+B \Sigma_{1}$.

In Section 6, we will see that, at least for the case of $E R A^{\omega}$, the transfer principles and induction in the system are close to optimal. When it comes to $P R A$, however, it seems worth mentioning another conservation result that can be obtained by the entirely different methods. Let $\left(I \Sigma_{1}^{s t}\right)$ denote the relativization of the schema of $\Sigma_{1}$ induction to the standard numbers. (Here too it does not hurt if we allow nonstandard parameters.) Let $N P R A^{\prime}$ consist of NPRA without the $\forall$-transfer axiom, together with $\left(I \Sigma_{1}^{s t}\right)$. Then we have the following:

THEOREM 4.5. If NPRA' proves $\forall^{s t} x \exists^{\text {st }} y \varphi(x, y)$ with $\varphi$ quantifier-free in the language of PRA, then PRA proves $\forall x \exists y \varphi(x, y)$.

Proof. The corresponding theorem for an intuitionistic version of $N P R A^{\prime}$ is proved in [7, Theorem 4.4]. By [7, Lemma 5.1], this intuitionstic theory proves that Markov's principle for primitive recursive relations holds on the standard numbers. Our $N P R A^{\prime}$ can therefore be interpreted in the intuitionistic version, using a double-negation translation.

Section 6 raises the question as to whether or not there is a common refinement of Theorems 2.2 and 4.5. Nonetheless, Theorem 4.5 is strong enough to yield the following celebrated result of Friedman. Here $W K L_{0}$ is essentially $W K L_{0}^{*}$ together with the schema of $\Sigma_{1}$ induction.

THEOREM 4.6. WKL $L_{0}$ is conservative over PRA for $\Pi_{2}$ sentences.

Proof. As in the proof of Theorem 4.4, $W K L_{0}$ is interpreted in NPRA'.

A further connection between Weak König's lemma and nonstandard analysis is discussed in [40].

One advantage to using an interpretation instead of a model-theoretic argument is that it becomes clear that the theorems can proved in weak theories. For example, let $S_{2}^{1}$ denote Buss' theory of feasible arithmetic (see $[10,13,15,18])$. Let $E R A^{*}$ denote the extension of $E R A$ with a function symbol for iterated exponentiation (a conservative extension of the theory sometimes denoted $E F A^{*}, I \Delta_{0}($ superexp $)$, or $I \Sigma_{0}+$ superexp; see [21]). 
THEOREM 4.7. $S_{2}^{1}$ proves that NERA $A^{\omega}$ is conservative over $E R A^{\omega}$, in the sense of Theorem 2.2. ERA* proves that NERA ${ }^{\omega}$ is conservative over ERA, in the sense of Theorem 2.2, and hence that NERA ${ }^{\omega}$ is consistent.

Proof. $S_{2}^{1}$ is strong enough to develop basic syntax, which is all that is needed to prove that the interpretation is sound. ERA* is strong enough to prove the cut-elimination and normalization theorems, which is enough to prove that $E R A^{\omega}$ is conservative over the quantifier-free version of $E R A$; and it is strong enough to define a truth predicate for quantifier-free formulae of $E R A$, which is enough to prove that ERA is consistent.

The formal system ERNA of $[37,38]$ can be interpreted in NERA, taking the variables of the former theory to range over the rational numbers of the latter, as in Section 5 below. Therefore, Theorem 4.7 refines the results of $[14,37,38]$.

Finally, let me mention, in passing, that these methods can be extended to stronger theories as well. With full Peano arithmetic, $P A$, one can take the nonstandard version, $N P A$, to include the transfer schema,

$$
s t\left(x_{1}\right) \wedge \ldots \wedge s t\left(x_{n}\right) \rightarrow\left(\varphi \leftrightarrow \varphi^{s t}\right),
$$

where $\varphi$ is any formula in the language of $P A$ with free variables $x_{1}, \ldots, x_{n}$; and the principle of standard induction,

$$
\varphi(0) \wedge \forall x(\varphi(x) \rightarrow \varphi(x+1)) \rightarrow \forall^{s t} x \varphi(x),
$$

for arbitrary formulae $\varphi$. By formalizing in $P A$ the usual construction of a nonstandard elementary extension of the natural numbers via compactness (more precisely, the construction of $\Sigma_{k}$ elementary extensions, for arbitrarily large $k$ ), one can show that $N P A$ is a conservative extension of $P A$. Another proof, using definable ultrapowers, is presented in [34]. But since the transfer principle above is equivalent to the assertion that the standard numbers are closed under suitable Skolem functions, with some additional work the forcing interpretation in Section 3 can be used to obtain yet another proof of this conservation result.

§5. Nonstandard analysis in weak theories. In this section I will sketch a way of developing parts of analysis in nonstandard theories of arithmetic like the ones discussed above. All of the definitions and theorems which follow should be thought of as taking place in $N E R A^{\omega}$. It is likely that similar methods can be used to carry out parts of analysis in $N P V^{\omega}$, but the situation there is more delicate; see the discussion in Section 6.

I will use the notation $\mathbb{N}^{*}$ to denote the nonstandard natural numbers, and interpret the type $\mathrm{N}$ of our nonstandard theories as ranging over $\mathbb{N}^{*}$. Hence, a quantifier of the form $\forall x \in \mathbb{N}^{*}$ denotes nothing more than quantification over the type $\mathrm{N}$ objects of the universe. On the other hand, I will let $\mathbb{N}$ denote the standard numbers, so $x \in \mathbb{N}$ means $s t(x)$, and a quantifier $\forall x \in \mathbb{N} \varphi$ is understood as $\forall x(s t(x) \rightarrow \varphi)$. 
The nonstandard integers, $\mathbb{Z}^{*}$, are defined to be ordered pairs $\langle a, b\rangle$ of nonstandard natural numbers, and the nonstandard rationals are defined to be ordered pairs $\langle a, b\rangle$ of nonstandard integers, with $b \neq 0$. The usual relations and arithmetic operations on integers and rationals, including $=\mathbb{Z}^{*},==_{\mathbb{Q}^{*}},<\mathbb{Z}^{*}$, $<_{\mathbb{Q}^{*}},+_{\mathbb{Z}^{*}},+_{\mathbb{Q}^{*}}, \ldots$ are defined in the usual way, and all are represented by type 1 terms in the language of $N E R A^{\omega}$. Note that equality is a defined relation on $\mathbb{Z}^{*}$ and $\mathbb{Q}^{*}$. An integer $\langle a, b\rangle$ is standard, written $\langle a, b\rangle \in \mathbb{Z}$, if it is equal to an integer $\langle c, d\rangle$, with $c, d \in \mathbb{N}$; or equivalently if $|a-b|$ is standard. Similarly, the standard rationals $\mathbb{Q}$ are those that are standard fractions when reduced to lowest terms. Note that the relations $x \in \mathbb{Z}$ and $x \in \mathbb{Q}$ are external, i.e. they are defined by formulae that involve the predicate st. As above, we can interpret quantification over $\mathbb{Z}^{*}, \mathbb{Z}, \mathbb{Q}^{*}$, and $\mathbb{Q}$ in the usual way; where notationally convenient I may write, for example, $\forall q^{\mathbb{Q}}$ instead of $\forall q \in \mathbb{Q}$. I will omit subscripts on operations like + and $\times$ when they are implicit in the context.

If $q$ is an element of $\mathbb{Q}^{*},\lfloor q\rfloor$ denotes the result of rounding $q$ down to the nearest integer and $\lceil q\rceil$ denotes the result of rounding up. A nonstandard rational $q$ is bounded if $\lceil q\rceil$ is standard and infinite otherwise. A nonstandard rational $q$ is infinitesimal if $q=0$ or $1 / q$ is infinite. Nonstandard rationals $q$ and $r$ are infinitely close, written $q \sim r$, if $q-r$ is infinitesimal. The properties of being bounded and of being an infinitesimal, and the relation of being infinitely close, are all external.

The standard real numbers, $\mathbb{R}$, are defined to be the bounded nonstandard rationals. If $r$ and $s$ are reals, $r=\mathbb{R} s$ means $r \sim s$. A function $f$ from $\mathbb{R}$ to $\mathbb{R}$ is simply a function $f$ from $\mathbb{Q}^{*}$ to $\mathbb{Q}^{*}$ preserving equality on the reals, that is, satisfying

$$
\forall r, s \in \mathbb{R}\left(r=_{\mathbb{R}} s \rightarrow f(r)=_{\mathbb{R}} f(s)\right) .
$$

One has to be careful in defining the ordering: $r<\mathbb{R}_{\mathbb{R}}$ means $r<\mathbb{Q}^{*} s \wedge r \not s$; $r \leq_{\mathbb{R}} s$ is equivalent to $r<\mathbb{Q}^{*} s \vee r \sim s$. If I have introduced $r$ and $s$ as real numbers, $r<s$ should be read as $r<\mathbb{R} s$; I will use $r<\mathbb{Q}^{*} s$ when I want to specify the order relation on nonstandard rationals. Note that unlike $=\mathbb{Q}^{*}$ and $<_{\mathbb{Q}^{*}}$, which are internal and, in fact, atomic relations, $=_{\mathbb{R}}$ and $<_{\mathbb{R}}$ are external.

The usual field operations on $\mathbb{Q}^{*}$ lift to make $\mathbb{R}$ an ordered field. Under this lifting, division by 0 can have unusual properties; for example, if $p$ is $1 / \omega$ and $q$ is $2 / \omega$, then, as real numbers, $p=q=0$, but $p / q=1 / 2$.

Let us pause for a moment to compare this to common developments of nonstandard analysis (as in, say, [20]). In such developments, one typically defines the nonstandard reals, $\mathbb{R}^{*}$, in which one can embed the standard ones; and any standard function $f$ from $\mathbb{R}$ to $\mathbb{R}$ has a nonstandard extension $f^{*}$ from $\mathbb{R}^{*}$ to $\mathbb{R}^{*}$. In our setup, nonstandard reals would have to be developed as type 1 objects, e.g. as Cauchy sequences of nonstandard rationals; general functions from $\mathbb{R}^{*} \rightarrow \mathbb{R}^{*}$ would then be type 2 . Here I propose to ignore the nonstandard reals entirely. We will see below that though this approach has 
some quirks, it suffices for the development of parts of real analysis, and it has the advantage that real numbers are represented by type 0 objects.

The following lemma says that one can bound the size of the numerator and denominator in the nonstandard representation of a real number.

Lemma 5.1. Let $x$ be an element of $\mathbb{R}$. Then there are $a \in \mathbb{Z}^{*}$ and $b \in \mathbb{N}^{*}$ such that $|a|<\omega, b<\omega$, and $x=_{\mathbb{R}} a / b$.

Proof. If $x=\mathbb{Q}^{*} 0$, take $a=0, b=1$. Otherwise, we can assume $x>\mathbb{Q}^{*} 0$; if $x<\mathbb{Q}^{*} 0$, apply the argument to $-x$.

Since $x \in \mathbb{R}, x$ is bounded by a standard natural number $c=\lceil x\rceil>0$. So if we let $b=\lfloor(\omega-1) / c\rfloor, b$ is nonstandard as well. We want to find $a$ such that

$$
\frac{a}{b} \leq \mathbb{Q}^{*} x<\mathbb{Q}^{*} \frac{a+1}{b}
$$

so let $a=\lfloor b x\rfloor$. Then $b<\omega$ and $a \leq{\mathbb{Q ^ { * }}} b x \leq\lfloor(\omega-1) / c\rfloor c \leq \omega-1<\omega$. Since $x-a / b<\mathbb{Q}^{*} 1 / b$, we have $x=\mathbb{R} a / b$, as needed.

The proof shows moreover that suitable values of $a$ and $b$ can be computed from $x$ by a type 1 term of $E R A^{\omega}$. The advantage bestowed by Lemma 5.1 is that certain quantifiers over the real numbers become equivalent to bounded ones. For example, suppose $\varphi(x)$ is a formula which respects equality of reals. Then $\forall x^{\mathbb{R}} \varphi(x)$ is equivalent to $\forall a^{\mathbb{N}^{*}}<\omega, b^{\mathbb{N}^{*}}<\omega(b \neq 0 \wedge s t(\lceil a / b\rceil) \rightarrow$ $\varphi( \pm a / b))$. This last formula is external, since it involves st. But if $\varphi$ is internal and one wants to quantify over a bounded range of real numbers, one can replace $s t(\lceil a / b\rceil)$ by an explicit bound, in which case the result is an internal formula. So, for example, if $R(x, y)$ is a relation (i.e. a type 1 term) in $N E R A^{\omega}$ that respects equality on the real numbers and $r$ and $s$ are reals, then $\forall x \in[r, s] R(x, y)$ is also equivalent to a relation in $N E R A^{\omega}$.

A function $f$ from $\mathbb{R}$ to $\mathbb{R}$ is continuous if it satisfies the usual $\varepsilon-\delta$ definition of continuity:

$$
\forall x^{\mathbb{R}} \forall \varepsilon^{\mathbb{R}}>0 \exists \delta^{\mathbb{R}}>0 \forall y^{\mathbb{R}}(|x-y|<\delta \rightarrow|f(x)-f(y)|<\varepsilon) .
$$

In $N E R A^{\omega}$ we have the following surprising fact:

Proposition 5.2. Every function $f \in \mathbb{R} \rightarrow \mathbb{R}$ is continuous.

Proof. Suppose we are given $f \in \mathbb{R} \rightarrow \mathbb{R}, x \in \mathbb{R}$, and $\varepsilon \in \mathbb{R}$ with $\varepsilon>\mathbb{R} 0$. It suffices to find a $\delta \in \mathbb{R}$ such that

$$
\forall h^{\mathbb{R}}(|h|<\delta \rightarrow|f(x+h)-f(x)|<\varepsilon) .
$$

Since $f$ respects equality on $\mathbb{R}$, we know that for each nonstandard natural number $m$,

(3) $\forall a^{\mathbb{Z}^{*}} \in(-\omega, \omega) \forall b<\omega\left(|a / b|<\mathbb{Q}^{*} 1 / m \rightarrow|f(x+a / b)-f(x)|<\mathbb{Q}^{*} \varepsilon / 2\right)$.

If (3) holds for all $m \geq 1$ let $m=0$, and otherwise, by induction, let $m$ be the greatest number less than $\omega$ such that (3) fails. Let $\delta=1 /(m+1)$. Since $m$ is standard, $\delta>\mathbb{R} 0$. 
I claim that this $\delta$ satisfies (2). Suppose $|h|<_{\mathbb{R}} \delta$. By Lemma $5.1, h=\mathbb{R} a / b$ for some $a \in \mathbb{Z}^{*}$ and $b \in \mathbb{N}^{*}$ with $|a|, b<\omega$. Then $|a / b|<\mathbb{Q}^{*} 1 /(m+1)$, and so $|f(x+a / b)-f(x)|<\mathbb{Q}^{*} \varepsilon / 2$. Since $f$ is a function on $\mathbb{R}$ and $h=\mathbb{R} a / b$, we have $|f(x+h)-f(x)| \leq_{\mathbb{R}} \varepsilon / 2<_{\mathbb{R}} \varepsilon$.

The proof above used induction on a bounded formula, and so does not go through in $N P V^{\omega}$. But in $N P V^{\omega}$ one can prove the converse, namely, that every function $f \in \mathbb{Q}^{*} \rightarrow \mathbb{Q}^{*}$ satisfying the continuity condition is in fact a function $f \in \mathbb{R} \rightarrow \mathbb{R}$.

At first glance, Proposition 5.2 seems blatantly false. After all, what about the function $f \in \mathbb{Q}^{*} \rightarrow \mathbb{Q}^{*}$ defined by

$$
f(x)= \begin{cases}0 & \text { if } x \leq \mathbb{Q}^{*} 0 \\ 1 & \text { otherwise }\end{cases}
$$

which is represented by a term of $N E R A^{\omega}$ ? The problem is that this is not a function from $\mathbb{R}$ to $\mathbb{R}$ : for example, $1 / \omega=\mathbb{R} 0$ but $f(1 / \omega) \neq \mathbb{R} f(0)$. On the other hand, the function $g \in \mathbb{Q}^{*} \rightarrow \mathbb{Q}^{*}$ defined by

$$
g(x)= \begin{cases}0 & \text { if } x \leq \mathbb{R} 0 \\ 1 & \text { otherwise }\end{cases}
$$

is not represented by a term of $N E R A^{\omega}$, since $x \leq_{\mathbb{R}} 0$ is external.

Thus, we have a development of analysis which, like Brouwer's, has the property that every well-defined function from $\mathbb{R}$ to $\mathbb{R}$ is continuous. This feature may help illuminate the Brouwerian world-view. What is going on is that in our framework, function variables $f, g, \ldots$ range over internal functions; and, in essence, Proposition 5.2 tells us that any function from $\mathbb{R} \rightarrow \mathbb{R}$ defined from an internal function from $\mathbb{Q}^{*} \rightarrow \mathbb{Q}^{*}$ is continuous. If one is loathe to give up functions like $g$ above, one can extend our theories with function variables ranging over external functions, which are not allowed to appear in the induction axioms. Thus, in a sense, Proposition 5.2 is not incompatible with classical mathematics; it only underscores the fact that, in the theory at hand, we have chosen to ignore the additional functions. For many purposes this restriction poses no great loss; for example, the function $f$ above is welldefined on any interval of $\mathbb{R}$ that does not contain 0 .

The examples that follow provide evidence that our framework allows a smooth development of elementary calculus.

TheOREM 5.3. If $f \in[0,1] \rightarrow \mathbb{R}$, then $f$ is uniformly continuous.

Proof. The proof is similar to that of Proposition 5.2 above.

Theorem 5.4 (Intermediate value theorem). Suppose $f \in[0,1] \rightarrow \mathbb{R}, f(0)=$ -1 , and $f(1)=1$. Then there is an $x \in[0,1]$ such that $f(x)=0$.

Proof. Considering $f$ as a function on $\mathbb{Q}^{*}$, let

$$
j=\max \left\{i<\omega \mid f(i / \omega)<\mathbb{Q}^{*} 0\right\}
$$


and let $x=j / \omega$. Since $j / \omega \sim(j+1) / \omega$, we have

$$
f((j+1) / \omega)=_{\mathbb{R}} f(j / \omega) \leq_{\mathbb{R}} 0 \leq_{\mathbb{R}} f((j+1) / \omega)
$$

and so $f(x)={ }_{\mathbb{R}} 0$.

Theorem 5.5 (Extreme value theorem). If $f \in[0,1] \rightarrow \mathbb{R}$, then $f$ attains a maximum value.

Proof. Again considering $f$ as a function on $\mathbb{Q}^{*}$, let

$$
y=\max _{0 \leq i \leq \omega} f(i / \omega),
$$

let $x=j / \omega$ satisfy $f(x)=\mathbb{Q}^{*} y$. That $y$ is a maximum is guaranteed by the fact that for any $x^{\prime} \in[0,1]$, there is an $i$ such that $x^{\prime} \sim i / \omega$.

Turning to differentiation, if $f \in \mathbb{R} \rightarrow \mathbb{R}$ and $x, y \in \mathbb{R}$, say $f^{\prime}(x)=y$ if

$$
\forall \varepsilon^{\mathbb{R}}>0 \exists \delta^{\mathbb{R}}>0 \forall h^{\mathbb{R}}\left(0 \neq_{\mathbb{R}}|h|<\delta \rightarrow\left|\frac{f(x+h)-f(x)}{h}-y\right|<\varepsilon\right) .
$$

This is not the strongest condition one can imagine, since it says nothing about the behavior of $f$ at nonzero infinitesimals. For example, it is possible that $f^{\prime}(0)=0$ while, as a function from $\mathbb{Q}^{*}$ to $\mathbb{Q}^{*}, f(x)$ oscillates between $-x$ and $x$ on an infinitesimal neighborhood of 0 . Say that $f^{\prime}(x)$ is strongly equal to $y$ if the formula above holds with $\neq_{\mathbb{R}}$ replaced by $\neq_{\mathbb{Q}}$.

Proposition 5.6. Let $f \in \mathbb{R} \rightarrow \mathbb{R}, x, y \in \mathbb{R}$. Then the following are equivalent:

1. $f^{\prime}(x)$ is strongly equal to $y$.

2. For every infinitesimal $h \neq \mathbb{Q}^{*} 0, \frac{f(x+h)-f(x)}{h} \sim y$.

If $f^{\prime}(x)$ is strongly equal to $y$, then $f^{\prime}(x)=y$.

Proof. The last claim is obvious. For the implication $1 \rightarrow 2$, suppose $f^{\prime}(x)$ is strongly equal to $y$ and let $h$ be a nonzero infinitesimal element of $\mathbb{Q}^{*}$. Then for every $\delta^{\mathbb{R}}>0,|h|<_{\mathbb{R}} \delta$. So for every standard $n,|(f(x+h)-f(x)) / h-y|<$ $1 / n$. This implies that $(f(x+h)-f(x)) / h-y$ is infinitesimal.

The proof that $2 \rightarrow 1$ is very similar to that of Proposition 5.2.

Corollary 5.7. Suppose $k$ is standard, and $f(x)=x^{k}$. Then for every $x$, $f^{\prime}(x)=k x^{k-1}$.

Proof. Suppose $h$ is infinitesimal. Calculating, we have

$$
\frac{(x+h)^{k}-x^{k}}{h}=k x^{k-1}+h\left[\sum_{i=2}^{k}\left(\begin{array}{c}
k \\
i
\end{array}\right) x^{k-i} h^{i-2}\right] .
$$

Using the facts that $k$ and $\lceil x\rceil$ are standard, it is not hard to show that the expression in brackets is standard, and so its product with $h$ is infinitesimal. 
One can continue, for example, by defining functions like $e^{x}$, sin $x$, and $\cos x$ using nonstandard finite segments of their Taylor series expansions, and then deriving their basic properties. For another example, there is an easy proof of the Cauchy-Peano theorem on the existence of solutions to differential equations, as described in [40]. There does not seem to be any bar to developing integral calculus in $N E R A^{\omega}$ in a similar manner.

§6. Notes and questions. This paper is a modest contribution to the study of weak theories of nonstandard arithmetic, and there are a number of questions and issues that need to be further explored. The questions discussed in this section fall into two groups: the first has to do with the metamathematical properties of the formal theories under consideration, and the second has to do with their utility with respect to the formal analysis of mathematics.

As far as the theories go, one would like to know the extent to which they are optimal, and whether or not they can be strengthened with additional principles of induction, transfer, and so on, while maintaining $\Pi_{2}$ conservativity. For example, we might want to strengthen the $\forall$-transfer axiom of Section 2 by allowing standard parameters: $\forall^{s t} \vec{y}\left(\forall^{s t} \vec{x} \varphi(\vec{x}, \vec{y}) \rightarrow \forall \vec{x} \varphi(\vec{x}, \vec{y})\right)$, where $\varphi$ is an internal $\Delta_{0}$ formula that does not involve $\omega$. The following shows that we cannot even add this mild strengthening to $N E R A^{\omega}$ without violating $\Pi_{2}$ conservativity:

Proposition 6.1. Over NERA $A^{\omega}, \forall$-transfer with parameters implies $\Sigma_{1}$ induction relative to the standard numbers, i.e. the schema

$$
\forall^{s t} \vec{y}\left(\psi(0, \vec{y}) \wedge \forall^{s t} u(\psi(u, \vec{y}) \rightarrow \psi(u+1, \vec{y})) \rightarrow \forall^{s t} u \psi(u, \vec{y})\right),
$$

for $\psi(u, \vec{y})$ of the form $\exists^{s t} x \varphi(u, x, \vec{y})$, where $\varphi$ is a $\Delta_{0}$ formula in the langauge of $E R A^{\omega}$.

Proof. $\forall$-transfer with parameters implies that if $\varphi(u, x, \vec{y})$ is as above and $u$ and $\vec{y}$ are assumed to be standard, then $\exists^{s t} x \varphi(u, x, \vec{y})$ is equivalent to $\exists x<\omega \varphi(u, x, \vec{y})$. By induction for bounded formulae in $N E R A^{\omega}$, if $\exists x<$ $\omega \varphi(u, x, \vec{y})$ fails for some $u$, there is a least such $u$; and is least $u$ has to be nonstandard.

This leaves open the question as to whether one can improve the conservation result for $N P R A^{\omega}$, using either the methods presented here or in [7]. How much transfer can one add? Can one add the unrelativized version of $\Sigma_{1}$ induction for formulae in the original language? In trying to strengthen the conservation result, one might make use of the fact that one can add $\Sigma_{1}$ induction, and even $\Pi_{2}$ collection, to $P R A^{\omega}$ without destroying $\Pi_{2}$ conservativity. Formalized or internalized versions of the various model-theoretic constructions presented in $[21,22,5]$ may also be useful in this regard.

Similarly, one can extend $P V$ with $\Sigma_{1}^{b}$ induction, yielding, essentially, Buss' theory $S_{2}^{1}$ (for various formulations, see [10, 13, 15, 18]). And one can extend $S_{2}^{1}$ with either a weak form of collection for arbitrary bounded formulae (see 
[11], or [19] for a simpler model-theoretic proof) or a stronger form of collection for $\Sigma_{2}^{b}$ formulae (see [13]). Can either of these results or the associated modeltheoretic constructions be used to strengthen the theory $N P V^{\omega}$ ? In particular, can one obtain a strengthening of $N P V^{\omega}$ that is strong enough to interpret the second-order theories of [18], which include a form of weak König's lemma?

The interpretation of Section 3 provides efficient translations between secondorder and higher-order systems; and the Dialectica interpretation [6, 15, 25, 43] can be used to interpret the higher type theories in their quantifier-free counterparts. But the interpretation does not work at the first-order level. By internalizing cut-elimination arguments, it seems that one should be able to interpret NPRA and NERA efficiently in PRA and ERA. Is there an efficient means of interpreting $N P V$ in $P V$ ? Or can one find specific counterexamples to show that this is not the case?

Is there a better way to treat equality in the theories presented here?

Are there interesting nonstandard versions of Feferman's theories of explicit mathematics?

There are more general questions, having to do with the formalization of mathematics in theories like the ones presented here. For example, what is required to formalize various parts of analysis? See [14, 17, 24, 25, 27, 28, 33, $35,37,38]$ for various approaches to answering this question. Do nonstandard theories provide a useful approach?

Can nonstandard theories like the ones presented here provide a perspicuous means of extracting polynomial bounds from proofs of theorems in analysis, as done by Kohlenbach [24, 25, 26, 27, 28]?

At the level of feasible computation, one would like to be able to formalize the study of feasible real analysis, as described in [23]. Fernandes and Ferreira [17] provides an initial step in this direction. Can nonstandard theories be used for this purpose as well?

What kind of theory of nonstandard arithmetic is sufficient to carry out the development of measure-theoretic probability, along the lines of Nelson [32]?

What is required for carrying out the nonstandard combinatorial arguments in proof complexity and circuit complexity in $[1,45,46]$ ?

Finally, some general methodological reflection is in order. As noted in the introduction, the motivations for formalizing mathematics in weak theories range from philosophical, to mathematical, to potential applications in computer science; and a variety of formal frameworks have been explored. To what extent to the various goals overlap, and are there general considerations that can help us evaluate the various frameworks? Under such an analysis, how do the nonstandard theories described here stack up?

Appendix A. The following example shows that one needs to be careful in stating conservation results for nonstandard theories. In particular:

- In the absence of transfer principles, the strength of the standard and nonstandard parts of the theory may differ. 
- Conservativity may not always be established in a weak theory.

- Conservativity does not guarantee the existence of an even remotely feasible translation.

Let $T$ be a weak theory of arithmetic, say ERA. Let $T^{\prime}$ be the extension of $T$ in a language with a new predicate symbol st, with axioms asserting the existence of a nonstandard number, plus all the axioms of Peano arithmetic relativized to st.

Proposition 6.2. $T^{\prime}$ is a conservative extension of $T$.

Proof. Any nonstandard model of $T$ can be expanded to a nonstandard model of $T^{\prime}$, by interpreting st as the standard numbers.

Indeed, the proof shows that Proposition 6.2 holds even if one adds arbitrary true arithmetic statements, relativized to st, to $T^{\prime}$.

Proposition 6.3. There is no $<\varepsilon_{0}$-recursive translation of proofs from $T^{\prime}$ to $T$.

Proof (SKETCh). If $d$ is a proof, let $|d|$ denote the length of $d$, according to the number of symbols. Let $\left\{F_{\alpha} \mid \alpha<\varepsilon_{0}\right\}$ denote the fast-growing hierarchy of functions up to $\varepsilon_{0}$, so every $<\varepsilon_{0}$-recursive function is eventually dominated by some such $F_{\alpha}$. For each $\alpha<\varepsilon_{0}$, Peano arithmetic proves the formalized $\Pi_{2}$ assertion $\forall x \exists y\left(F_{\alpha}(x)=y\right)$. So, for each natural number $n$, there is a short proof of $\exists y\left(F_{\alpha}(\bar{n})=y\right)$ in $P A$. Since the axioms of $P A$ hold relativized to $s t$ in $T^{\prime}$, for each natural number $n$ there is a short proof of $\exists^{s t} y\left(F_{\alpha}(\bar{n})=y\right)$ in $T^{\prime}$, and hence a short proof of $\exists y\left(F_{\alpha}(\bar{n})=y\right)$.

On the other hand, any proof of $\exists y\left(F_{\alpha}(\bar{n})=y\right)$ in $T$ has to be long. For, given any proof $d$ of $\exists y\left(F_{\alpha}(\bar{n})=y\right)$, by Herbrand's theorem one can extract a term $t$ of $E R A$ such that $F_{\alpha}(\bar{n})=t$ holds, where the length of $t$, and hence its value, can be bounded by a fixed primitive recursive function of $|d|$. So, for $\alpha$ large, $|d|$ has to be relatively close to $F_{\alpha}(n)$.

Put more precisely: given $\alpha$, one can find a primitive recursive function $f$, such that for each $n$ there is a proof of $\exists y\left(F_{\alpha}(\bar{n})=y\right)$ in $T^{\prime}$ of length at most $f(n)$. At the same time, there is a fixed primitive recursive function $g$ such that if $d$ is any proof of $\exists y\left(F_{\alpha}(\bar{n})=y\right)$ in $T$, then $g(|d|) \geq F_{\alpha}(n)$. So, infinitely often, the translation maps a proof of length $m$ to a proof of length at least $g^{-1}\left(F_{\alpha}\left(f^{-1}(m)\right)\right)$. But given any $<\varepsilon_{0}$-recursive function $h$, one can choose $\alpha$ large enough so that for any primitive recursive $f$ and $g, h$ is eventually dominated by this last function.

Appendix B. If one is not concerned with an ultimate reduction to classical logic, the forcing relation can be simplified somewhat. To start with, we can restrict our attention to conditions that do not force falsity, i.e. define the relation $\operatorname{Cond}(p)$ to hold if and only if $p \forall \perp$. This modification requires some changes to the proofs in Section 3. For example, now conditions $p$ and $q$ may be incomparable, which is to say, $p \wedge q$ may not be a condition; the 
forcing clause for implication, $p \Vdash \varphi \rightarrow \psi$, becomes equivalent to the assertion $\forall q(q \Vdash \varphi \wedge \operatorname{Cond}(p \wedge q) \rightarrow p \wedge q \Vdash \psi)$. Also, clause 1 of Lemma 3.9 must be modified to read "for each quantifier-free internal formula $\varphi$ of $L^{s t}, P R A^{\omega}$ proves $\operatorname{Cond}(\widehat{\varphi}) \rightarrow(\widehat{\varphi} \Vdash \varphi)$."

The advantage to modifying the notion of a condition in this way that forcing for negation, disjunction, and existential quantification can be expressed more easily:

- $p \Vdash \neg \varphi$ if and only if $\forall q \preceq p(q \forall \varphi)$

- $p \Vdash \varphi \vee \psi$ if and only if $\forall q \preceq p \exists r \preceq q(r \Vdash \varphi$ or $r \Vdash \psi)$

- $p \Vdash \exists x \varphi$ if and only if $\forall q \preceq p \exists r \preceq q \exists \tilde{x}(r \Vdash \varphi)$

Here all the quantifiers are taken to be implicitly relativized to conditions, that is, predicates satisfying Cond. The definion of forcing for a doubly-negated formula becomes equivalent to the usual notion of generic validity:

- $p \Vdash \neg \neg \varphi$ if and only if $\forall q \preceq p \exists r \preceq q(r \Vdash \varphi)$

Lemma 3.7 then asserts that if $\varphi$ is generically valid below $p$, it is forced by $p$. The reader can check that using the equivalences above one can finish off the proof of Theorem 2.2 without the help of Lemma 3.15.

Further inspection of the proofs in Section 3 shows that when it comes to using the conditions $\forall^{s t} u \forall v p(u, v, \omega)$, the universal quantifer over $v$ is no longer needed, and the universal quantifier over standard $u$ is only used in the context $\forall^{s t} u(\widehat{t} \nless u)$, expressing that $t$ denotes a nonstandard element. We can more frugally take conditions to be represented pairs of the form $\langle\alpha, f\rangle$, where $\alpha$ is a predicate on $\mathrm{N}$ (represented by its characteristic function) and $f$ is a function from $\mathrm{N}$ to $\mathrm{N}$. Define such a pair $\langle\alpha, f\rangle$ to be a condition if it satisfies

$$
\forall z \exists \omega(\alpha(\omega) \wedge f(\omega) \geq z)
$$

expressing that the predicate $\alpha$ holds of values of $\omega$ making $f$ arbitrarily large. The relation $\langle\beta, g\rangle \preceq\langle\alpha, f\rangle$ is defined by

$$
\langle\beta, g\rangle \preceq\langle\alpha, f\rangle \equiv \forall \omega(\beta(\omega) \rightarrow \alpha(\omega) \wedge g(\omega) \leq f(\omega)),
$$

and if $\langle\alpha, f\rangle$ and $\langle\beta, g\rangle$ are compatible conditions, then their greatest lower bound is given by $\langle\alpha \wedge \beta, \min (f, g)\rangle$. The forcing clauses for atomic formulae are now as follows:

- $p \Vdash t_{1}=t_{2} \equiv \exists z \forall \omega\left(\alpha(\omega) \wedge f(\omega) \geq z \rightarrow \widehat{t}_{1}=\widehat{t}_{2}\right)$

- $p \Vdash t_{1}<t_{2} \equiv \exists z \forall \omega\left(\alpha(\omega) \wedge f(\omega) \geq z \rightarrow \widehat{t}_{1}<\widehat{t}_{2}\right)$

- $p \Vdash s t(t) \equiv \exists z \forall \omega(\alpha(\omega) \wedge f(\omega) \geq z \rightarrow \widehat{t}<z)$

We then have $\langle\top, \widehat{t}\rangle \Vdash \neg s t(t)$; in particular, if $i d$ is the identity function, $\langle T, i d\rangle$ forces that $\omega$ is nonstandard. The reader can verify that the proof of Theorem 2.2 still goes through, mutatis mutandis.

Finally, in the case of $N P R A^{\omega}$, the translation can be simplified even further, provided one allows $\Sigma_{1}$ induction in the target theory. Conditions can be taken 
to be unary predicates $p$ on $\mathrm{N}$ that hold for infinitely many values of $\omega$,

$$
\forall z \exists \omega \geq z p(\omega),
$$

corresponding to sets in the Fréchet filter. These are the forcing clauses for atomic formulae:

- $p \Vdash t_{1}=t_{2} \equiv \exists z \forall \omega \geq z\left(p(\omega) \rightarrow \widehat{t}_{1}=\widehat{t}_{2}\right)$

- $p \Vdash t_{1}<t_{2} \equiv \exists z \forall \omega \geq z\left(p(\omega) \rightarrow \widehat{t}_{1}<\widehat{t}_{2}\right)$

- $p \Vdash s t(t) \equiv \exists z \forall \omega \geq z(p(\omega) \rightarrow \widehat{t}<z)$

In other words, $p$ forces $t_{1}=t_{2}$ if and only if $\widehat{t}_{1}=\widehat{t}_{2}$ holds for all but finitely many values of $\omega$ satisfying $p$. Although the necessity of having $\Sigma_{1}$ induction in the interpreting theory weakens the result, it is perhaps surprising that such a straightfoward translation can be used to interpret nonstandard reasoning.

$\Sigma_{1}$ induction is required to verify that $\neg \neg s t(t) \rightarrow s t(t)$ is forced, as follows.

Lemma 6.4. Over PRA $A^{\omega}, \Sigma_{1}$ induction is equivalent to the following principle:

$$
\exists z \forall y(f(y) \leq z) \rightarrow \exists x \forall y(f(y) \leq f(x)) .
$$

This principle expresses the fact that every bounded function on $\mathrm{N}$ has a least upper bound, and attains it. Only the forward direction of the lemma is required below, though the equivalence seems interesting in its own right.

Proof. The contrapositive of the principle is equivalent to

$$
\forall x \exists y(f(y)>f(x)) \rightarrow \forall z \exists y(f(y)>z) .
$$

We will show that $\Sigma_{1}$ induction is equivalent to (4), arguing in $P R A^{\omega}$. For the forward direction, suppose $\forall x \exists y(f(y)>f(x))$. By induction on $z$, it is easy to show $\exists y(f(y)>z)$.

Conversely, suppose $\varphi(u, v)$ is a $\Delta_{0}$ formula satisfying the two hypotheses of $\Sigma_{1}$ induction, $\exists v \varphi(0, v)$ and $\forall u(\exists v \varphi(u, v) \rightarrow \exists v \varphi(u+1, v))$. We need to show $\forall u \exists v \varphi(u, v)$. Define $f(x)$ to be the greatest $w \leq x$ such that $\forall u<$ $w \exists v \leq x \varphi(u, v)$. It is not hard to show that for every $x, \exists y(f(y)>f(x))$; if $f(x)$ is 0 , this follows from the first hypothesis, and otherwise it follows from the second. By (4), for every $u$ there is a $y$ such that $f(y)>u$; the definition of $f$ implies $\exists v \varphi(u, v)$.

LemMa 6.5. Let $t$ be any term. With the modified definition of forcing, $P R A^{\omega}+\Sigma_{1}$ induction proves the following: Let $p$ be any condition and let $q$ be the predicate defined by

$$
q(\omega) \equiv p(\omega) \wedge \forall u<\omega(p(u) \rightarrow \widehat{t}(u)<\widehat{t}(\omega)) .
$$

Then if $q$ is a condition, $q \Vdash \neg s t(t)$.

Proof. The idea is that $q$ corresponds to a subset of $p$ on which $\widehat{t}$ is strictly increasing as a function of $\omega$. Suppose $q$ is a condition, and let $r$ be any predicate satisfying $r \preceq q$. It suffices to show that if $r \Vdash s t(t)$, then $r$ is not a condition. 
So, suppose $r \Vdash s t(t)$, i.e.

$$
\exists z \forall \omega \geq z(r(\omega) \rightarrow \widehat{t}(\omega)<z) .
$$

Since $r \preceq q$ we know that $\widehat{t}$ is increasing on $r$, that is,

$$
\forall u, v(r(u) \wedge r(v) \wedge u<v \rightarrow \widehat{t}(u)<\widehat{t}(v)) .
$$

Define $f$ by

$$
f(v)=\max _{u \leq v \wedge r(u)} \widehat{t}(u) .
$$

By (5) we have that $f$ is bounded by $z$. Using the principle of Lemma 6.4, there is a value $u$ such that $\forall v(f(v) \leq f(u))$. But then (6) implies

$$
\forall \omega>u \neg r(\omega),
$$

so $r$ is not a condition.

Lemma 6.6. $P R A^{\omega}+\Sigma_{1}$ induction proves that $\neg \neg s t(t) \rightarrow s t(t)$ is forced.

Proof. Suppose $p \Vdash \neg \neg s t(t)$. Then $\forall q \preceq p(q \not \neg s t(t))$. Define $q$ as in the statement of Lemma 6.5. Then $q \preceq p$, and if $q$ is a condition then $q \Vdash \neg s t(t)$; so $q$ is not a condition. This means we have $\exists z \forall \omega \geq z \neg q(\omega)$, i.e. for some $z$ we have

$$
\forall \omega \geq z(p(\omega) \rightarrow \exists u<\omega(p(u) \wedge \widehat{t}(\omega) \leq \widehat{t}(u)) .
$$

Since $p$ is a condition, we can pick an $\omega \geq z$ satisfying $p(\omega)$, and let $v=$ $\max _{u \leq \omega \wedge p(u)} \widehat{t}(u)$. Then we have

$$
\forall \omega \geq z(p(\omega) \rightarrow t(\omega) \leq v)
$$

which implies $p \Vdash s t(t)$.

With the modified forcing definition, it is easy to show that $\neg s t(\omega)$ is forced. So, in the end, we can conclude that whenever $N P R A^{\omega}$ proves a formula $\varphi$, $P R A^{\omega}+\Sigma_{1}$ induction proves that $\varphi$ is forced.

\section{REFERENCES}

[1] Miklos Ajtai, The complexity of the pigeonhole principle, Proceedings of the IEEE 29th annual symposium on foundations of computer science, 1988, pp. 346-355.

[2] Jeremy Avigad, Formalizing forcing arguments in subsystems of second-order arithmetic, Annals of Pure and Applied Logic, vol. 82 (1996), no. 2, pp. 165-191.

[3] - Interpreting classical theories in constructive ones, To appear in the Journal of Symbolic Logic.

[4] - Ordinal analysis without proofs, to appear in Reflections: a collection honoring Solomon Feferman on his 70th birthday, AK Peters.

[5] - Saturated models of universal theories, submitted.

[6] Jeremy Avigad and Solomon Feferman, Gödel's functional (Dialectica) interpretation, in Buss [12].

[7] Jeremy Avigad and Jeffrey Helzner, Transfer principles for intuitionistic nonstandard arithmetic, submitted. 
[8] Jon Barwise (editor), The handbook of mathematical logic, North-Holland, Amsterdam, 1977.

[9] Michael Beeson, Foundations of constructive mathematics, Springer, Berlin, 1985.

[10] Samuel Buss, Bounded arithmetic, Bibliopolis, Naples, 1986, A reprinting of the author's 1985 Princeton University dissertation.

[11] - A conservation result concerning bounded theories and the collection axiom,

Proceedings of the American Mathematical Society, vol. 100 (1987), pp. 709-716.

[12] - (editor), The handbook of proof theory, North-Holland, Amsterdam, 1998.

[13] — First-order proof theory of arithmetic, in Buss [12].

[14] Rolando Chuaqui and Patrick Suppes, Free-variable axiomatic foundations of infinitesimal analysis: a fragment with finitary consistency proof, The Journal of Symbolic Logic, vol. 60 (1995), no. 1, pp. 122-159.

[15] Stephen CoOK and Alasdair URquhart, Functional interpretations of feasibly constructive arithmetic, Annals of Pure and Applied Logic, vol. 63 (1993), pp. 103-200.

[16] Solomon Feferman, Theories of finite type related to mathematical practice, in Barwise [8], pp. 913-971.

[17] A. M. Fernandes and Fernando Ferreira, Groundwork for weak analysis, Draft.

[18] Fernando Ferreira, A feasible theory for analysis, The Journal of Symbolic Logic, vol. 59 (1994), pp. 1001-1011.

[19] — A note on a result of Buss concerning bounded theories and the collection scheme, Portugaliae Mathematica, vol. 52 (1995), no. 3, pp. 331-336.

[20] ROBERT GOLDBLATT, Lectures on the hyperreals: an introduction to nonstandard analysis, Springer, New York, 1998.

[21] Petr HÁJeK and PAvel Pudlák, Metamathematics of first-order arithmetic, Springer, Berlin, 1993.

[22] Richard Kaye, Models of Peano arithmetic, Clarendon, Oxford, 1991.

[23] Ker-I Ko, Complexity theory of real functions, Birkhäuser, Boston, 1991.

[24] UlRICH KoHLENBACH, Effective moduli from ineffective uniqueness proofs: an unwinding of de La Vallée Poussin's proof for Chebycheff approximation, Annals of Pure and Applied Logic, vol. 64 (1993), pp. 27-94.

[25] - Analyzing proofs in analysis, Logic: From foundations to applications: European logic colloquium '93 (W. Hodges et al., editors), Clarendon Press, Oxford, 1996, pp. 225-260.

[26] — Mathematically strong subsystems of analysis with low rate of growth of provably recursive functionals, Archive for Mathematical Logic, vol. 36 (1996), pp. 3171.

$[27]-$, Proof theory and computational analysis, Third workshop on computation and approximation (Birmingham, 1997), Elsevier, Amsterdam, 1998, p. 34 pp. (electronic).

[28] - Foundational and mathematical uses of higher types, to appear in Reflections: a collection honoring Solomon Feferman on his 70th birthday, AK Peters.

[29] Georg Kreisel, Axiomatizations of nonstandard analysis that are conservative extensions of classical systems of analysis, Applications of model theory to algebra, analysis, and probability (W. A. J. Luxemburg, editor), Holt, Rinehart, and Winston, 1967, pp. 93-106.

[30] Ieke MoerdiJk and Erik Palmgren, Minimal models of Heyting arithmetic, The Journal of Symbolic Logic, vol. 62 (1997), pp. 1448-1460.

[31] EDWARD NELSON, Internal set theory: a new approach to nonstandard analysis, Bulletin of the American Mathematics Society, vol. 83 (1977), pp. 1165-1198.

$[32] —$, Radically elementary probability theory, Princeton University, 1987. 
[33] Erik Palmgren, Developments in constructive nonstandard analysis, The Bulletin of Symbolic Logic, vol. 4 (1998), pp. 233-272.

[34] — An effective conservation result for nonstandard analysis, Mathematical Logic Quarterly, vol. 46 (2000), pp. 17-23.

[35] Stephen Simpson, Subsystems of second-order arithmetic, Springer, Berlin, 1998.

[36] Stephen Simpson and Rick Smith, Factorization of polynomials and $\Sigma_{1}^{0}$ induction, Annals of Pure and Applied Logic, vol. 31 (1986), pp. 289-306.

[37] Richard Sommer and Patrick Suppes, Finite models of elementary recursive nonstandard analysis, Notas De la Sociedad de Matematica de Chile, vol. 15 (1996), no. 1.

[38] Richard Sommer and Patrick Suppes, Dispensing with the continuum, J. Math. Psych., vol. 41 (1997), no. 1, pp. 3-10.

[39] Thomas Strahm, Theories with self-application and computational complexity, Draft.

[40] KaZuyuki Tanaka, Non-standard analysis in $W K L_{0}$, Mathematical Logic Quarterly, vol. 43 (1997), pp. 401-412.

[41] A. S. Troelstra, Metamathematical investigation of intuitionistic arithmetic and analysis, Lecture Notes in Mathematics \#344, Springer, Berlin, 1973.

[42] — Aspects of constructive mathematics, in Barwise [8], pp. 973-1052.

[43] - Introductory note to 1958 and 1972, Kurt Gödel: Collected works (Solomon Feferman et al. editors), vol. II, Oxford University Press, New York, 1990, pp. 217241.

[44] - Realizability, in Buss [12].

[45] Alex Wilkie, Modèles non standard de l'arithmétique, et complexité algorithmique, Modèles non standard en arithmétique et théorie des ensembles, Publications Mathémathique de l'Université Paris VII, vol. 22, 1985.

[46] Alan Woods, Approximating the structures accepted by a constant depth circuit or satisfying a sentence - a nonstandard approach, Logic and random structures, Amer. Math. Soc., Providence, RI, 1997, pp. 109-130.

E-mail: avigad@cmu.edu, http://www.andrew.cmu.edu/ avigad

DEPARTMENT OF PHILOSOPHY, CARNEGIE MELLON UNIVERSITY, PITTSBURGH, PA 15213, USA 